\title{
Fate of Light Scalar Mesons 1
}

\author{
N.N. Achasov \\ Sobolev Institute for Mathematics, 630090 Novosibirsk, Russia \\ email: achasov@math.nsc.ru
}

\begin{abstract}
It is shown that all predictions for the light scalars, based on their four-quark nature, are supported by experiment. The future research program is outlined also.

PACS numbers: PACS numbers: 12.39.-x, 13.40.-f, 13.60. Le, 13.75. Lb
\end{abstract}

\section{Introduction}

\section{OVERTURE}

Peter Higgs, C. R. Physique 8 (2007) 970-972:

"Another example, which comes closer to the kind of symmetry breaking which is of interest in particle physics, is superfluidity. In 1947 Bogoliubov studied Bose condensation of an infinite system of neutral spinless bosons with short-range repulsive two-body interactions. Such a condensate is characterised by a 'macroscopic wave function' (the order parameter) which is complex; its modulus squared is a measure of the observable condensate density, but its argument (which is unobservable) is arbitrary, thus breaking the symmetry of the dynamics under rotations of the boson wave functions in the Argand diagram. The short-range interactions are represented in the second-quantised Hamiltonian by a term proportional to the square of the particle density, that is, to a quartic in the components of the scalar quantum field."

See also, N.N. Achasov, Physics of Particles and Nuclei, 2010, Vol. 41, No. 6, pp. 891-895. DOI: 10.1134/S1063779610060134, arXiv:1001.3468 [hep-ph].

\section{Outline}

The $a_{0}(980)$ and $f_{0}(980)$ mesons are well-established parts of the proposed light scalar meson nonet [1]. From the beginning, the $a_{0}(980)$ and $f_{0}(980)$ mesons became one of the central problems of nonperturbative QCD, as they are important for understanding the way chiral symmetry is realized in the low-energy region and, consequently, for understanding confinement. Many experimental and theoretical papers have been devoted to this subject. There is much evidence that supports the four-quark model of light scalar mesons [2, 3].

\footnotetext{
${ }^{1}$ Invited talk at the International Conference "Problems of Theoretical and Mathematical Physics" dedicated to the 110th anniversary of the birth of the outstanding Russian scientist - mathematician and physicist Nikolai Nikolaevich Bogolyubov, JINR, Dubna, September 11 $13,2019$.
} 
The suppression of the $a_{0}^{0}(980)$ and $f_{0}(980)$ resonances in the $\gamma \gamma \rightarrow \eta \pi^{0}$ and $\gamma \gamma \rightarrow \pi \pi$ reactions, respectively, was predicted in 1982 [4], $\Gamma_{a_{0}^{0} \gamma \gamma} \approx \Gamma_{f_{0} \gamma \gamma} \approx 0.27 \mathrm{keV}$, and confirmed by experiment [1].

The high quality Belle data [5], Fig. 1, 2, allowed to elucidate the mechanisms of the $\sigma(600), f_{0}(980)$, and $a_{0}^{0}(980)$ resonance production in $\gamma \gamma$ collisions confirmed their four-quark structure. $\sigma(600)=f_{0}(500)$ !

Light scalar mesons are produced in $\gamma \gamma$ collisions via rescatterings, mainly via the $\gamma \gamma \rightarrow \pi^{+} \pi^{-} \rightarrow f_{0}(500), \gamma \gamma \rightarrow K^{+} K^{-} \rightarrow f_{0}(980) / a_{0}$ (980 transitions, that is, via the four-quark transitions. As for $a_{2}(1320)$ and $f_{2}(1270)$ (the well-known $q \bar{q}$ states), they are produced mainly via the two-quark transitions (direct couplings with $\gamma \gamma$ ) [6, 7, 8, 9].

As a result the practically model-independent prediction of the $q \bar{q}$ model $g_{f_{2} \gamma \gamma}^{2}: g_{a_{2} \gamma \gamma}^{2}=$ 25 : 9 agrees with experiment rather well. As to the ideal $q \bar{q}$ model prediction $g_{f_{0} \gamma \gamma}^{2}$ : $g_{a_{0} \gamma \gamma}^{2}=25: 9$, it is excluded by experiment.

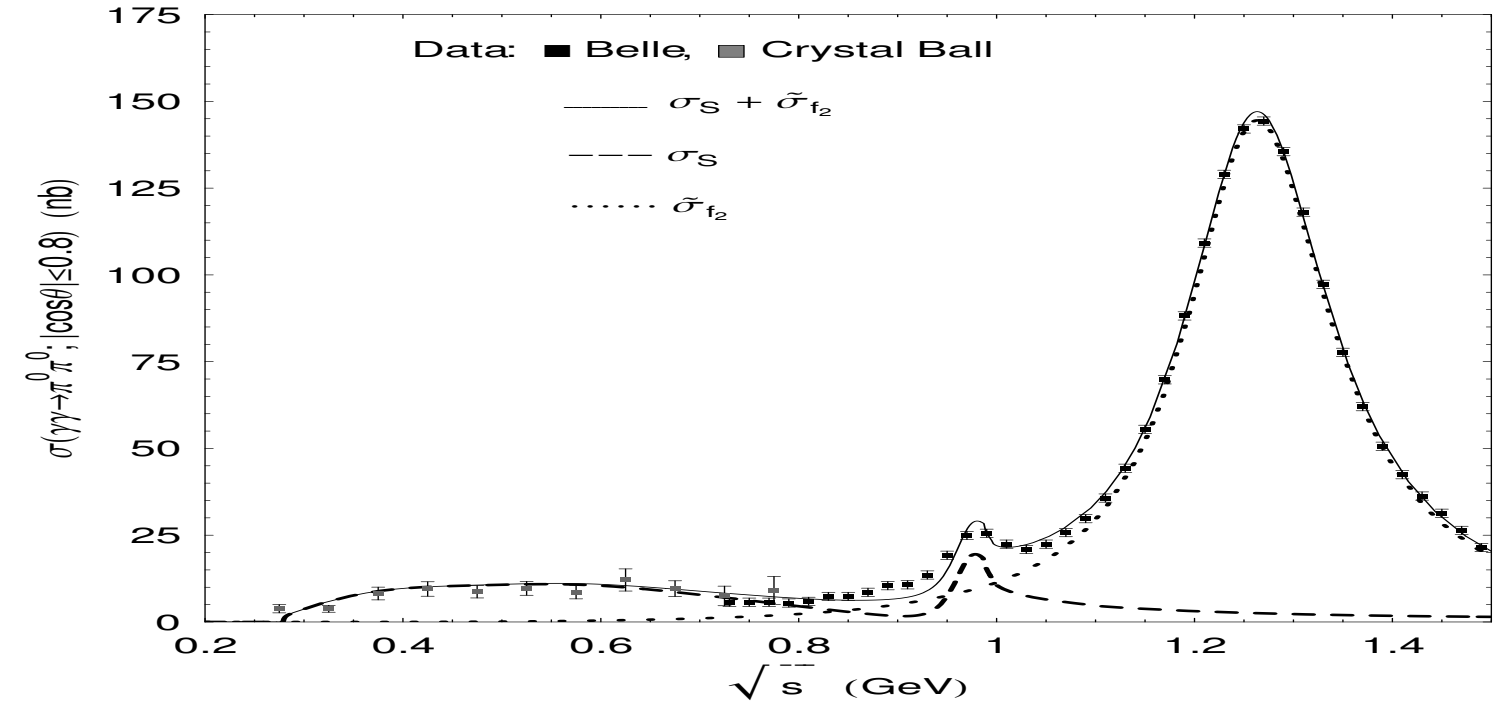

Figure 1: The Belle data [5]. Our fit from Ref. [6]

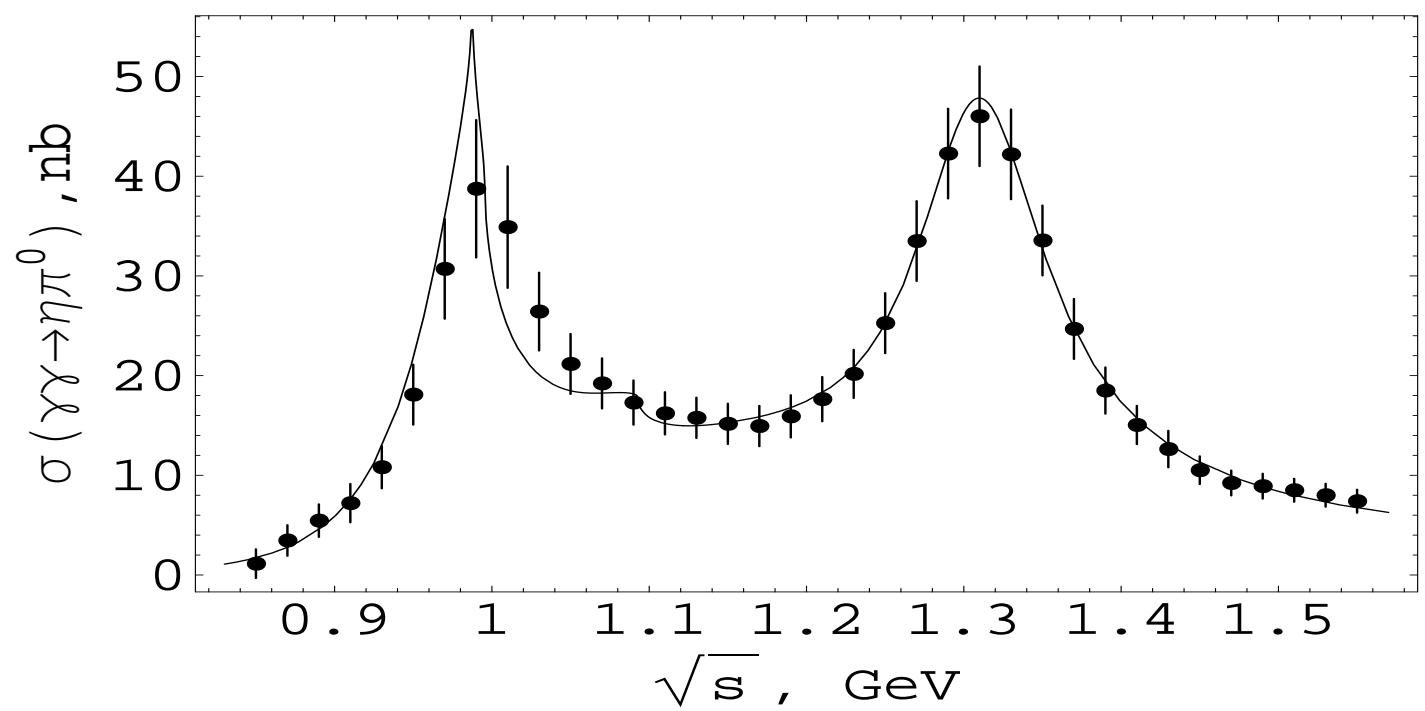

Figure 2: The Belle date [5]. Our fit from Ref. [9]. 
Note also that the absence of $J / \psi \rightarrow \gamma f_{0}(980), \rho a_{0}(980), \omega f_{0}(980)$ decays in the presence of the intense $J / \psi \rightarrow \gamma f_{2}(1270), \gamma f_{2}^{\prime}(1525)$, $\rho a_{2}(1320), \omega f_{2}(1270)$ decays is at variance with the $P$-wave two-quark, $q \bar{q}$, structure of $a_{0}(980)$ and $f_{0}(980)$ resonances [10, 11].

The argument in favor of the four-quark nature of $a_{0}(980)$ and $f_{0}(980)$ is the fact that the $\phi(1020) \rightarrow a_{0}^{0}(980) \gamma$ and $\phi(1020) \rightarrow f_{0}(980) \gamma$ decays go through the kaon loop: $\phi \rightarrow K^{+} K^{-} \rightarrow a_{0}^{0}(980) \gamma, \phi \rightarrow K^{+} K^{-} \rightarrow f_{0}(980) \gamma$, i.e., via the four-quark transition [12, 13, 14, 15, 16, 17, 18, 9].

The kaon-loop model was suggested in Ref. [12] and confirmed by experiment ten years later [19, 20, 21, 22], Figs. 3, 4.

In Ref. [16] it was shown that the production of $a_{0}^{0}(980)$ and $f_{0}(980)$ in $\phi \rightarrow a_{0}^{0}(980) \gamma \rightarrow$ $\eta \pi^{0} \gamma$ and $\phi \rightarrow f_{0}(980) \gamma \rightarrow \pi^{0} \pi^{0} \gamma$ decays is caused by the four-quark transitions, resulting in strong restrictions on the large $N_{C}$ expansion of the decay amplitudes. The analysis showed that these constraints give new evidence in favor of the four-quark nature of the $a_{0}(980)$ and $f_{0}(980)$ mesons.

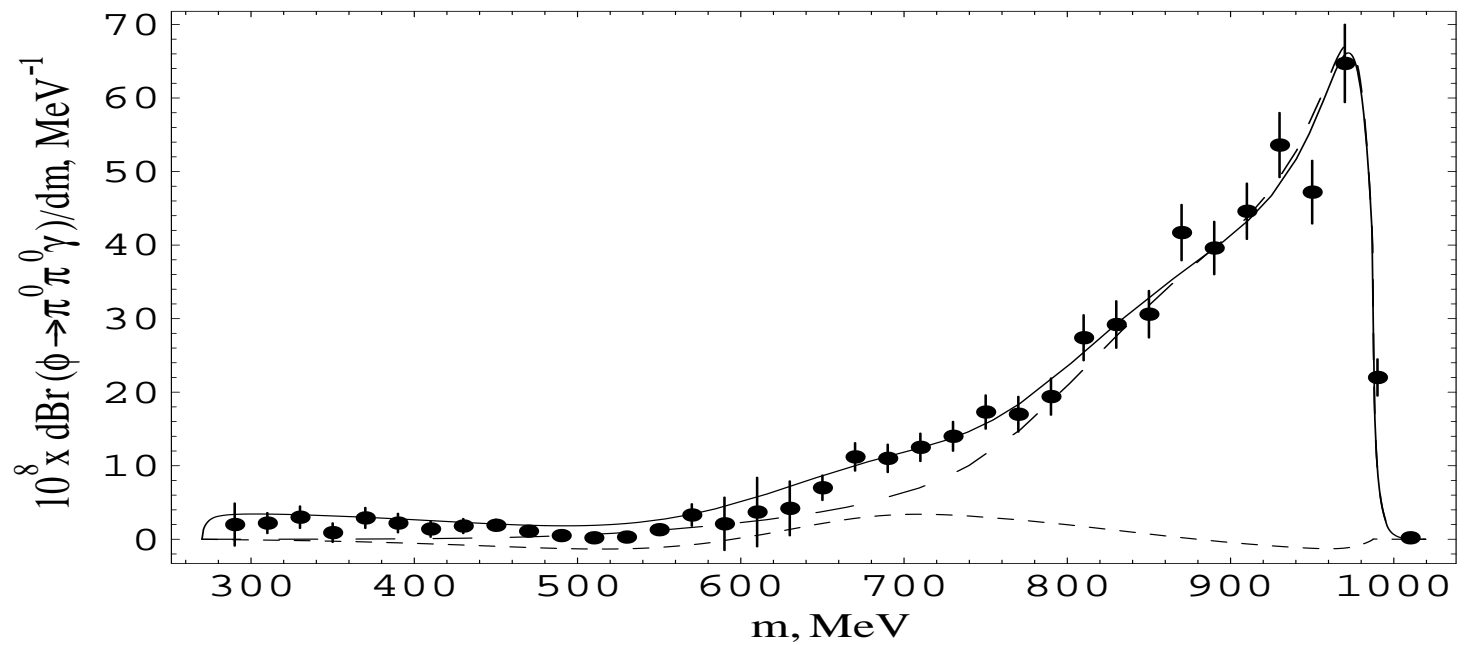

Figure 3: The KLOE data [21]. Our fit Ref. [18].

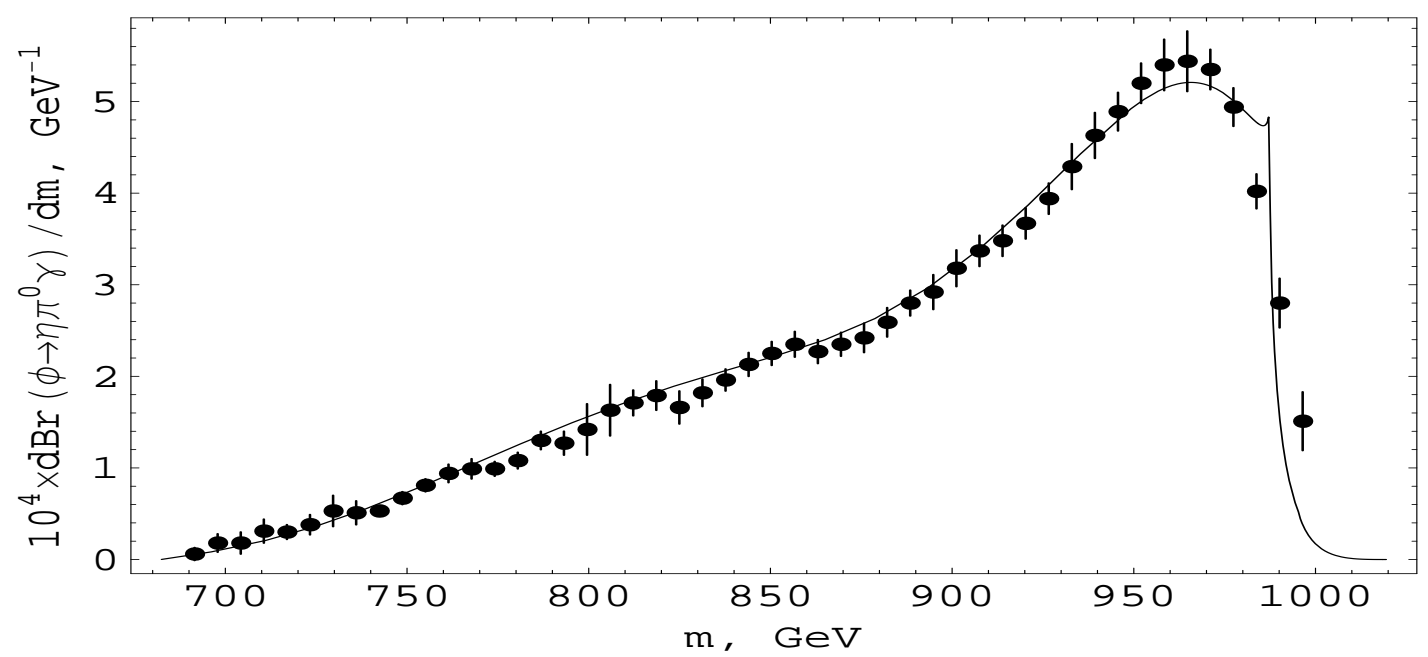

Figure 4: The KLOE data [22]. Our fit from Ref. [9]. 
In Refs. [23, 24] it was shown that the description of the $\phi \rightarrow K^{+} K^{-} \rightarrow \gamma a_{0}^{0}(980) / f_{0}(980)$ decays requires virtual momenta of $K(\bar{K})$ greater than $2 \mathrm{GeV}$, while in the case of loose molecules with a binding energy about $20 \mathrm{MeV}$, they would have to be about $100 \mathrm{MeV}$. Besides, it should be noted that the production of scalar mesons in the pion-nucleon collisions with large momentum transfers also points to their compactness [25].

In Refs. [26] it was also shown that the linear $S_{L}(2) \times S_{R}(2) \sigma$ model [27] contains a chiral shielding of the $\sigma$ meson and reflects all of the main features of low energy $\pi \pi \rightarrow \pi \pi$ and $\gamma \gamma \rightarrow \pi \pi$ reactions up to energy $0.8 \mathrm{GeV}$ and agrees with the four-quark nature of the $\sigma$ meson.

This allowed for the development of a phenomenological model with the right analytical properties in the complex $s$ plane that took into account the linear $\sigma$ model, the $\sigma(600)-$ $f_{0}(980)$ mixing and the background [28].

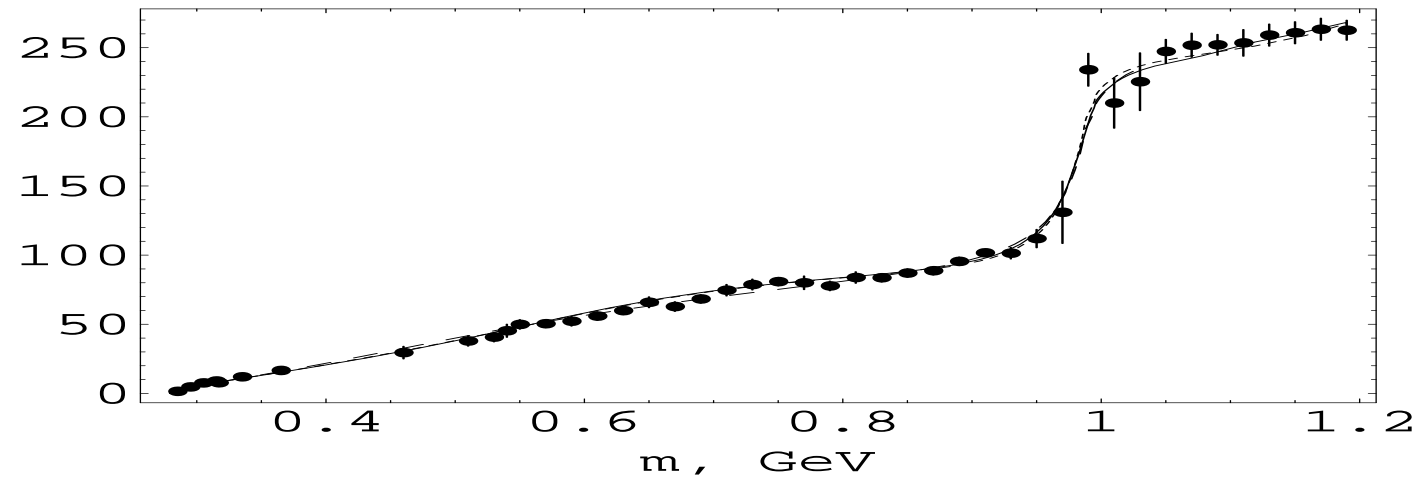

Figure 5: The phase $\delta_{0}^{0}$ of the $\pi \pi$ scattering (degrees) is shown.

This background has a left cut inspired by crossing symmetry, and the resulting amplitude agrees with results obtained using the chiral expansion, dispersion relations, and the Roy equation [29] and with the four-quark nature of the $\sigma(600)$ and $f_{0}(980)$ mesons as well. This model well describes the experimental data on $\pi \pi \rightarrow \pi \pi$ scattering up to $1.2 \mathrm{GeV}$, Fig. 5 .

In Refs. [9, 30] It is shown that the recent data on the $K_{S}^{0} K^{+}$correlation in $\mathrm{Pb}-\mathrm{Pb}$ interactions Ref. [31], Fig. 6, agree with the data on the $\gamma \gamma \rightarrow \eta \pi^{0}$ and $\phi \rightarrow \eta \pi^{0} \gamma$ reactions and support the four-quark model of the $a_{0}(980)$ meson. It is shown that the data does not contradict the validity of the Gaussian assumption.

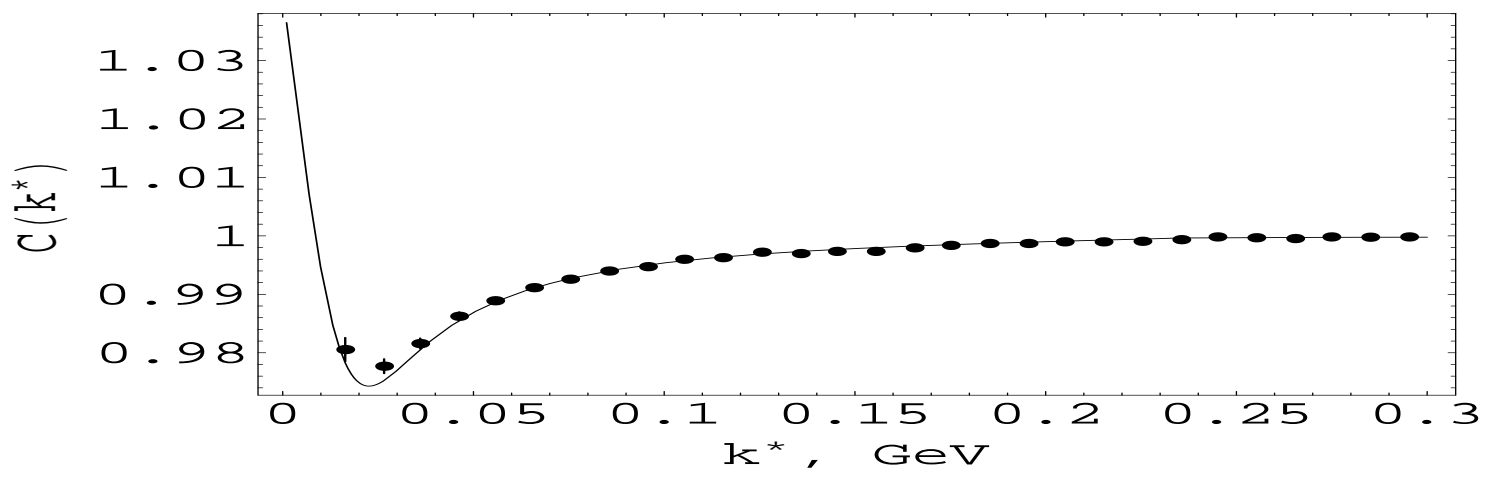

Figure 6: The $K_{s}^{0} K^{+}$correlation $C(k *), k^{*}$ is the kaon momentum in the kaon pair rest frame,see Refs. [9, 30] 


\section{Outlook}

In Refs. [32, 33] it was suggested the program of studying light scalars in semileptonic $D$ and $B$ decays, which are the unique probe of the $q \bar{q}$ constituent pair in the light scalars. We studied the CLEO data about production of scalars $\sigma(600)$ and $f_{0}(980)$ in the $D_{s}^{+} \rightarrow s \bar{s} e^{+} \nu_{e} \rightarrow \pi^{+} \pi^{-} e^{+} \nu$ decays, Fig. 7 . The conclusion was that the fraction of the $s \bar{s}$ constituent components in $\sigma(600)$ and $f_{0}(980)$ is small. Unfortunately, the CLEO statistics [34] is rather poor, Fig. 8, and thus new high-statistics data are highly desirable.

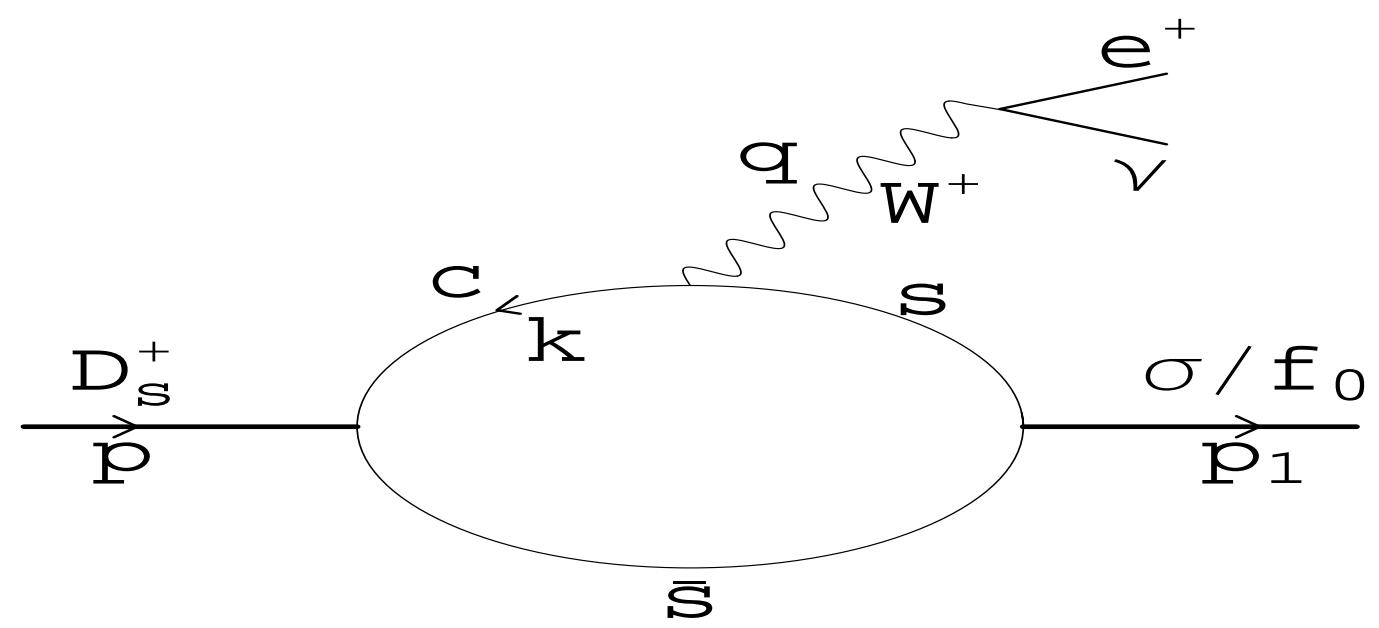

Figure 7: The mechanisms of the $D_{s}^{+} \rightarrow \sigma / f_{0} e^{+} \nu$ decays.

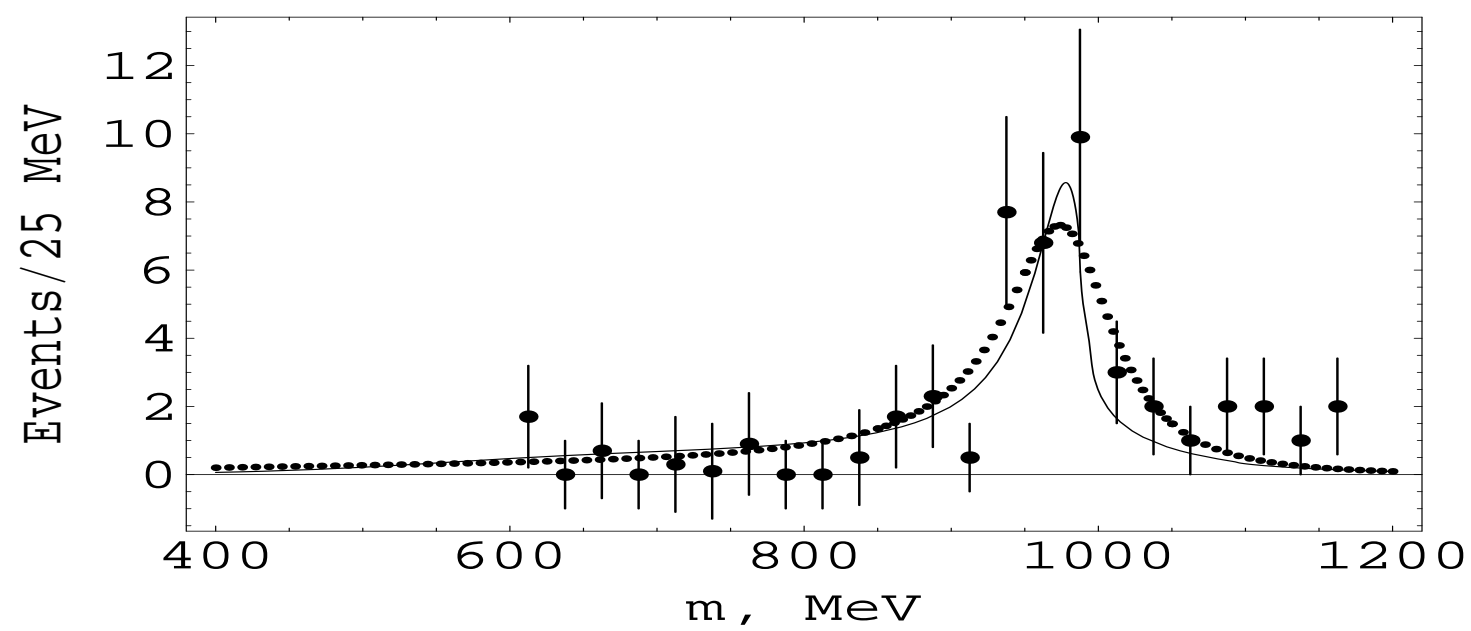

Figure 8: The CLEO data on the invariant $\pi^{+} \pi^{-}$mass $(m)$ distribution for $D_{s}^{+} \rightarrow$ $\pi^{+} \pi^{-} e^{+} \nu$ decay. The dotted line is the fit from CLEO. Our theoretical curve is the solid line.

In Refs. [32, 33] it was noted that no less interesting is the study of semileptonic decays of $D^{0}$ and $D^{+}$mesons:

$D^{+} \rightarrow d \bar{d} e^{+} \nu_{e} \rightarrow\left[\sigma(600)+f_{0}(980)\right] e^{+} \nu_{e} \rightarrow \pi^{+} \pi^{-} e^{+} \nu_{e}$,

$D^{0} \rightarrow d \bar{u} e^{+} \nu_{e} \rightarrow a_{0}^{-} e^{+} \nu_{e} \rightarrow \pi^{-} \eta e^{+} \nu_{e}$ and 
$D^{+} \rightarrow d \bar{d} e^{+} \nu_{e} \rightarrow a_{0}^{0} e^{+} \nu_{e} \rightarrow \pi^{0} \eta e^{+} \nu_{e}$

or the charged-conjugated ones which had not been investigated.

It is tempting to study light scalar mesons in semileptonic decays of $B$ mesons [33]:

$B^{0} \rightarrow d \bar{u} e^{+} \nu_{e} \rightarrow a_{0}^{-} e^{+} \nu_{e} \rightarrow \pi^{-} \eta e^{+} \nu_{e}$,

$B^{+} \rightarrow u \bar{u} e^{+} \nu_{e} \rightarrow a_{0}^{0} e^{+} \nu_{e} \rightarrow \pi^{0} \eta e^{+} \nu_{e}$ and

$B^{+} \rightarrow u \bar{u} e^{+} \nu_{e} \rightarrow\left[\sigma(600)+f_{0}(980)\right] e^{+} \nu_{e} \rightarrow \pi^{+} \pi^{-} e^{+} \nu_{e}$

or the charged-conjugated ones.

Recently BESIII Collaboration measured the decays $D^{0} \rightarrow d \bar{u} e^{+} \nu \rightarrow a_{0}^{-} e^{+} \nu \rightarrow$ $\pi^{-} \eta e^{+} \nu$ and $D^{+} \rightarrow d \bar{d} e^{+} \nu \rightarrow a_{0}^{0} e^{+} \nu \rightarrow \pi^{0} \eta e^{+} \nu$ for the first time [35].

In Ref. [9] we discuss these measurements taking into account also contribution of $a_{0}^{\prime}$ meson with mass about $1400 \mathrm{MeV}$, Fig. 9 and Fig. 12 .

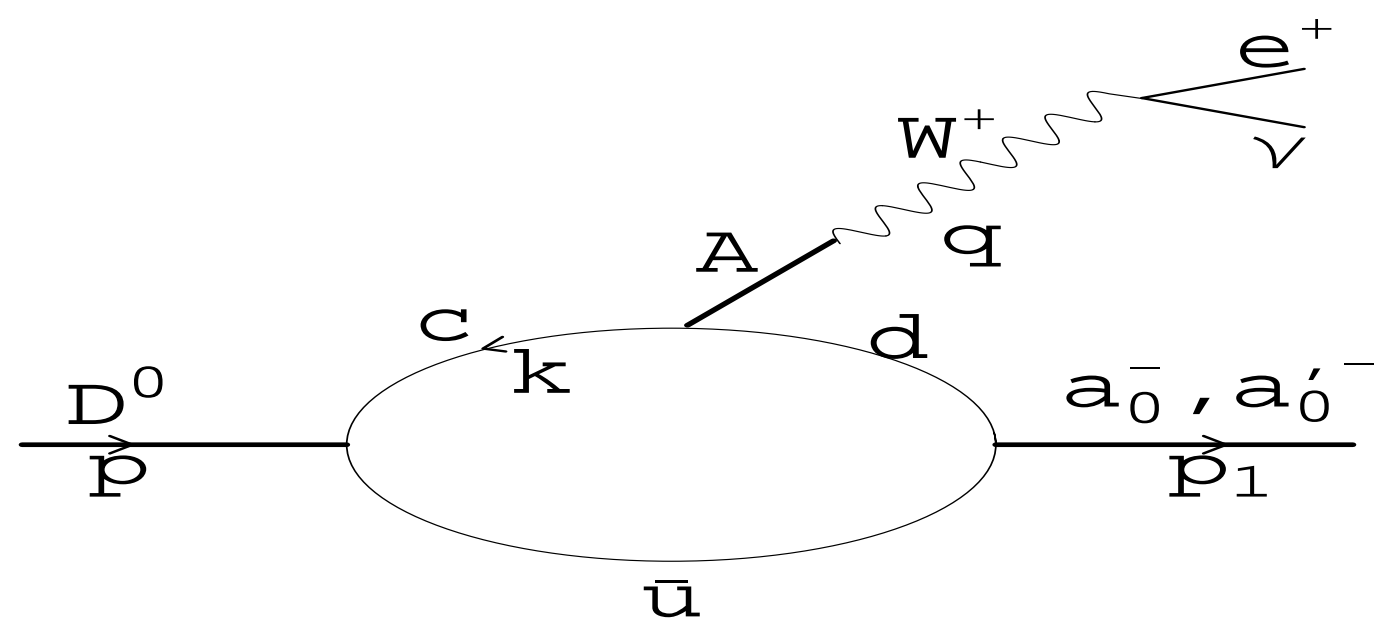

Figure 9: The mechanisms of the $D^{0} \rightarrow d \bar{u} e^{+} \nu_{e} \rightarrow a_{0}^{-}, a_{0}^{\prime-} e^{+} \nu_{e}$ decays.

Below, Figs. 10, 11, is presented a variant when $a_{0}^{-}(980)$ has no $q \bar{q}$ constituent component at all, that is, $a_{0}^{-}(980)$ is produced as a result of mixing $a_{0}^{\prime-}(1400) \rightarrow a_{0}^{-}(980)$, $D^{0} \rightarrow d \bar{u} e^{+} \nu_{e} \rightarrow a_{0}^{\prime-} e^{+} \nu_{e} \rightarrow a_{0}^{-} e^{+} \nu_{e} \rightarrow \pi^{-} \eta e^{+} \nu_{e}$.

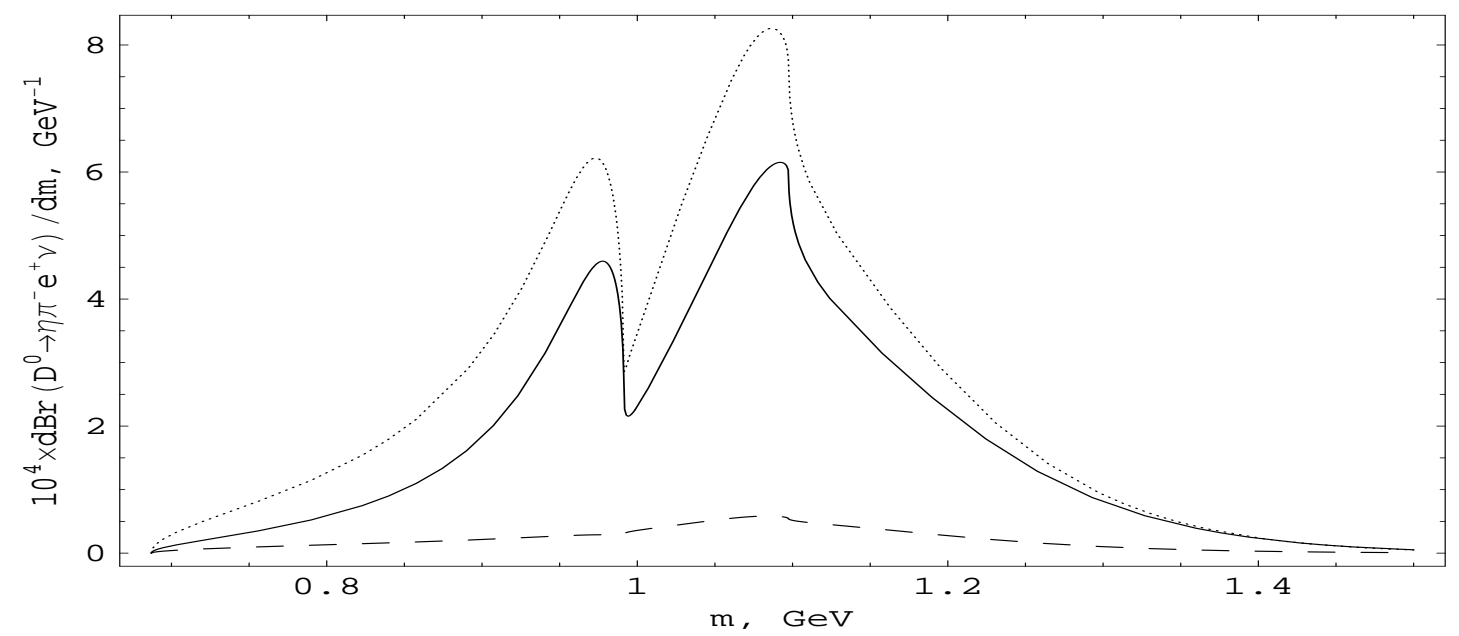

Figure 10: The $D^{0} \rightarrow d \bar{u} e^{+} \nu_{e} \rightarrow a_{0}^{\prime-} e^{+} \nu_{e} \rightarrow a_{0}^{-} e^{+} \nu_{e} \rightarrow \pi^{-} \eta e^{+} \nu_{e}$ spectrum. 


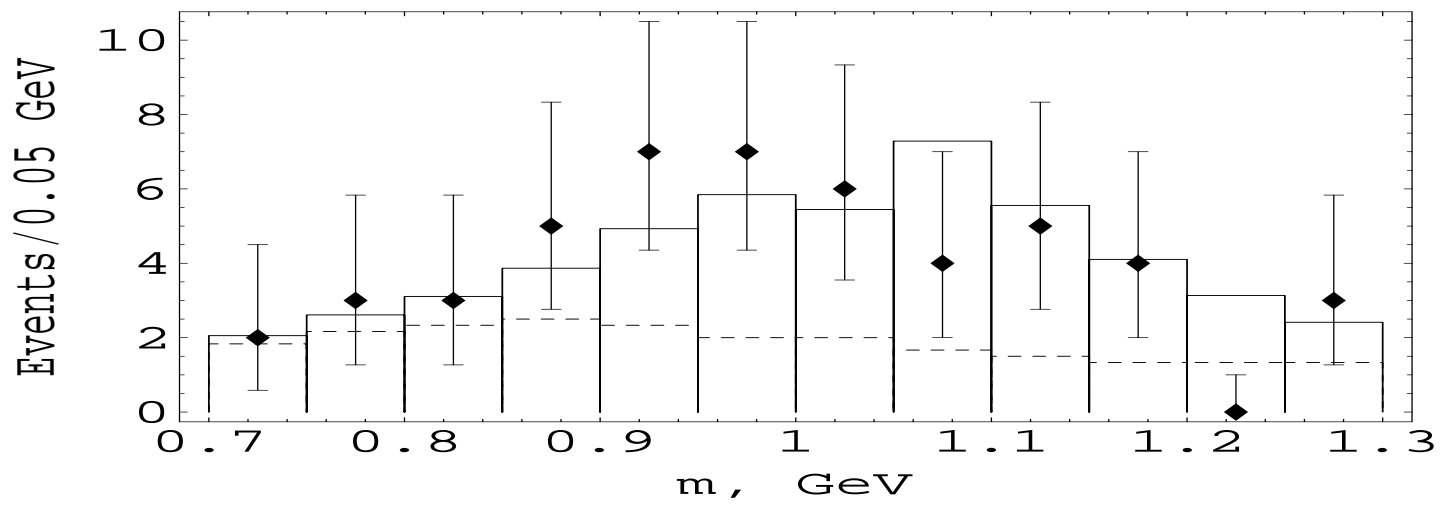

Figure 11: The $D^{0} \rightarrow d \bar{u} e^{+} \nu_{e} \rightarrow a_{0}^{\prime-} e^{+} \nu_{e} \rightarrow a_{0}^{-} e^{+} \nu_{e} \rightarrow \pi^{-} \eta e^{+} \nu_{e}$ spectrum.

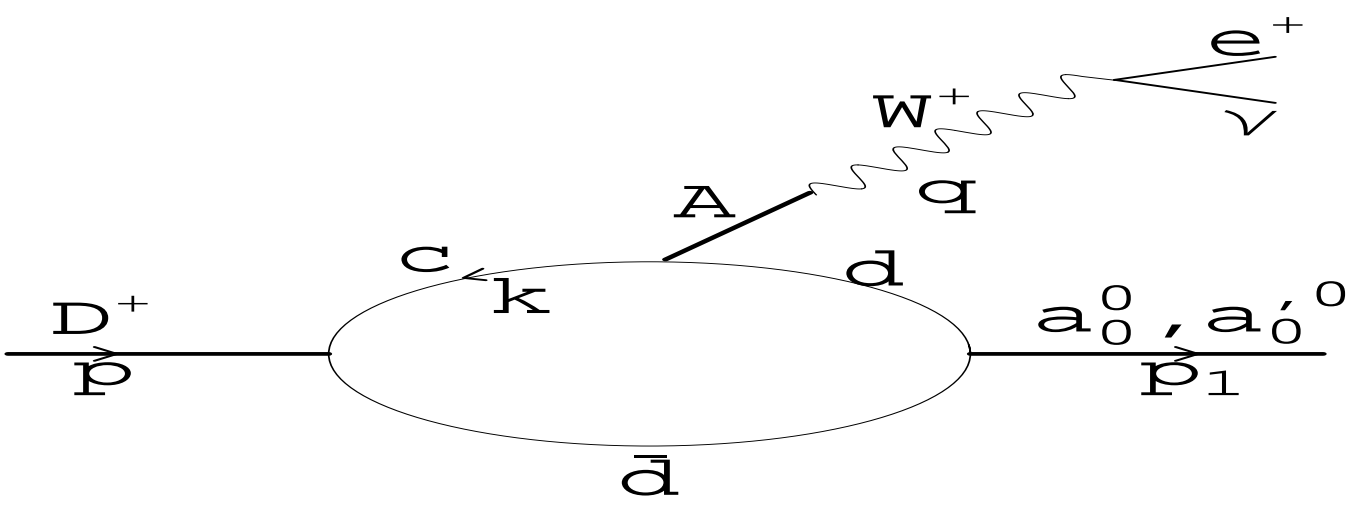

Figure 12: The mechanisms of the $D^{+} \rightarrow d \bar{d} e^{+} \nu_{e} \rightarrow a_{0}^{0}, a_{0}^{\prime 0} e^{+} \nu_{e}$ decays.

Below, Figs. 13, 14, is presented a variant when $a_{0}^{0}(980)$ has no $q \bar{q}$ constituent component at all, that is, $a_{0}^{0}(980)$ is produced as a result of mixing $a_{0}^{\prime 0}(1400) \rightarrow a_{0}^{0}(980)$, $D^{+} \rightarrow d \bar{d} e^{+} \nu_{e} \rightarrow a_{0}^{\prime 0} e^{+} \nu_{e} \rightarrow a_{0}^{0} e^{+} \nu_{e} \rightarrow \pi^{0} \eta e^{+} \nu_{e}$.

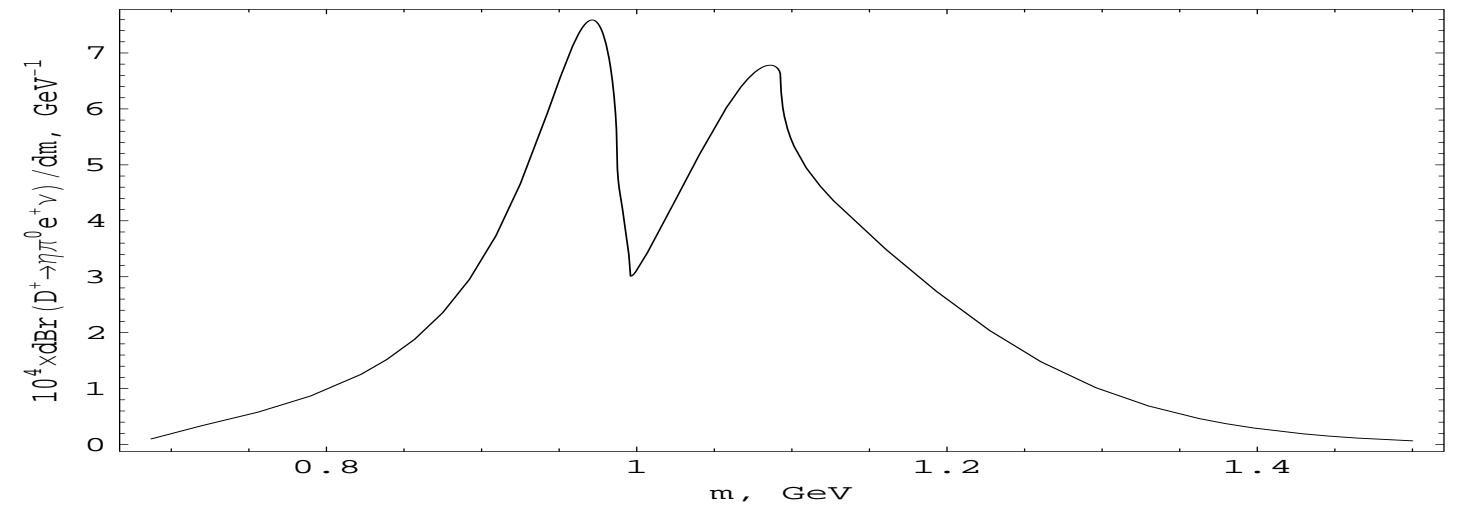

Figure 13: The $D^{+} \rightarrow d \bar{d} e^{+} \nu_{e} \rightarrow a_{0}^{\prime 0} e^{+} \nu_{e} \rightarrow a_{0}^{0} e^{+} \nu_{e} \rightarrow \pi^{0} \eta e^{+} \nu_{e}$ spectrum. 


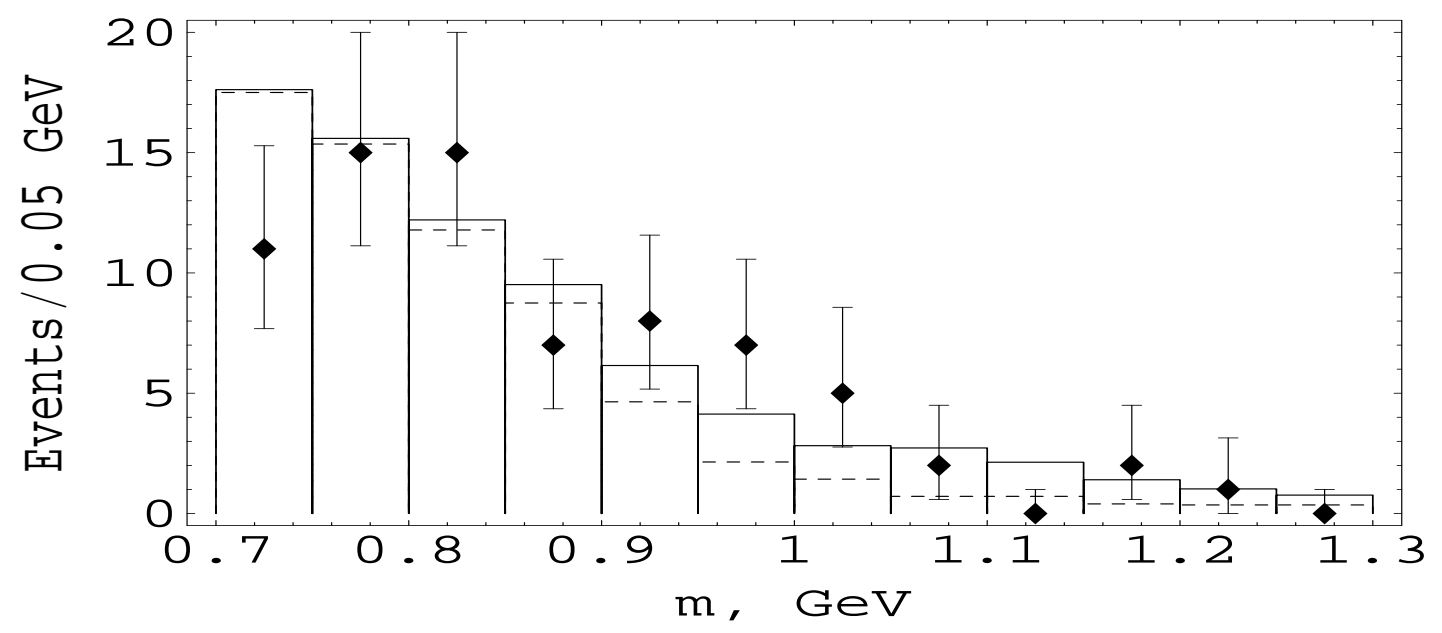

Figure 14: The $D^{+} \rightarrow d \bar{d} e^{+} \nu_{e} \rightarrow a_{0}^{\prime 0} e^{+} \nu_{e} \rightarrow a_{0}^{0} e^{+} \nu_{e} \rightarrow \pi^{0} \eta e^{+} \nu_{e}$ spectrum.

The first measurements of BESIII directly indicate the absence of the constituent $d \bar{d}$ and $d \bar{u}$ pairs in the $a_{0}^{0}(980)$ and $a_{0}^{-}(980)$ states respectively. But the the present the statistics is not adequate for the conclusions about details of the $\pi \eta$ production.

\section{Acknowledgements}

I am grateful Organizers for the kind Invitation.

I am grateful to A.V. Kiselev and G.N. Shestakov for collaboration.

The work was supported by the program of fundamental scientific researches of the SB RAS No. II.15.1., project No. 0314-2019-0021.

\section{References}

[1] Tanabashi M. etal. (Particle Data Group)// Phys. Rev. D. 2018. V. 98. P. 030001.

[2] Jaffe R.L.// Phys. Rev. D. 1977. V. 15. P. 267. P. 281.

[3] Weinberg S. // Phys. Rev. Lett. 2013. V. 110. P. 261601.

[4] Achasov N.N., Devyanin S.A. and Shestakov G.N.// Phys. Lett. B. 1982. V. 108. P. 134; Achasov N.N. Devyanin S.A. and Shestakov G.N.// Z. Phys. C. 1982. V. 16. P. 55.

[5] Mori T. et al.// Phys. Rev. D. 2007. V. 75. P. 051101; Uehara S. et al.// Phys. Rev. D. 2008. V. 78. P. 052004; Uehara S. et al.// Phys. Rev. D. 2009. V. 80. P. 032001.

[6] Achasov N.N. and Shestakov G.N.// Phys. Rev. D. 2008. V. 77. P. 074020.

[7] Achasov N.N. and Shestakov G.N.// Phys. Rev. D. 2010. V. 81. P. 094029.

[8] Achasov N.N. and Shestakov G.N.// Phys.Usp. 2011. V. 54. P. 799.

[9] Achasov N.N. and A.V. Kiselev A.V.// Phys. Rev. D 2018.V. 98. P. 096009.

[10] Achasov N.N.// Phys. Usp. 1998. V. 41. P. 1149.

[11] Achasov N.N.// Phys. At. Nucl. 2002. V. 65. P. 546.

[12] Achasov N.N. and V.N. Ivanchenko V.N.// Nucl. Phys. B. 1989. V. 315. P. 465.

[13] Achasov N.N. and Gubin V.V.// Phys.Rev. D. 1997. V. 56. P.4084. 
[14] Achasov N.N. and Gubin V.V.// Phys. Rev. D. 2001. V. 63. P.094007.

[15] Achasov N.N. and Kiselev A.V.// Phys. Rev. D. 2003.V. 68. P. 014006.

[16] Achasov N.N.// Nucl. Phys. A. 2003. V. 728. P. 425.

[17] Achasov N.N. and Kiselev A.V.// Phys. Rev. D. 2006. V. 73. P. 054029.

[18] Achasov N.N. and Kiselev A.V.// Phys. Rev. D. 2012. V. 85. P. 094016.

[19] Achasov M.N. et al. (SND Collaboration)// Phys. Lett. B. 1998. V. 438. P. 441; Achasov M.N. et al. (SND Collaboration) Phys.Lett. B. 1998. V. 440, P. 442;

Achasov M.N. et al. (SND Collaboration)// Phys. Lett. B. 2000. V. 479. P. 53; Achasov M.N. et al. (SND Collaboration)// Phys. Lett. B. 2000. V. 485. P. 349.

[20] Akhmetshin R.R. et al. (CMD-2 Collaboration)// Phys. Lett. B. 1999. V. 462. P. 380.

[21] Aloisio A. et al. (KLOE Collaboration)// Phys. Lett. B. 2002. V. 537, P. 21.

[22] Ambrosino F. et al. (KLOE Collaboration)// Phys. Lett. B. 2009. V. 681. P. 5.

[23] Achasov N.N. and Kiselev A.V.// Phys. Rev. D. 2007. V. 76. P. 077501; Achasov N.N. and Kiselev A.V. Phys. Rev. D. (2008). V.78. P. 058502.

[24] Achasov N.N., Gubin V.V. and Shevchenko V.I.// Phys. Rev. D. 1997. V. 56. P. 203.

[25] Achasov N.N. and Shestakov G.N.// Phys. Rev. D. 1998. V. 58. P. 054011.

[26] Achasov N.N. and Shestakov G.N.// Phys. Rev. D. 1994. V. 49. P. 5779; Achasov N.N. and Shestakov G.N.// Phys. Rev. Lett. 2007. V. 99. P. 072001.

[27] Gell-Mann M. and Levy M.// Nuovo Cim. 1960. V. 16. P.705.

[28] Achasov N.N. and Kiselev A.V.// Phys. Rev. D. 2011. V. 83. P. 054008; Achasov N.N. and Kiselev A.V.// Phys. Rev. D. 2012. V. 85. P. 094016.

[29] Caprini I., G. Colangelo G., and H. Leutwyler H.// Phys. Rev. Lett. 2006. V. 96. P. 132001.

[30] Achasov N.N. and Kiselev A.V.// Phys. Rev. D. 2018. V. 97. P. 036015.

[31] S. Acharya S. et al. (ALICE Collaboration)// Phys. Lett. B. 2017. v. 774. p. 64.

[32] Achasov N.N. and Kiselev A.V.// Phys. Rev. D. 2012. V. 86. P. 114010.

[33] Achasov N.N. and Kiselev A.V.// Int. J. Mod. Phys.: Conference Series. 2014. V. 35. P. 1460447.

[34] Ecklund K.M. et al. (CLEO Collaboration)// Phys. Rev. D. 2009. V. 80. P. 052009.

[35] M. Ablikim M. et al. (BESIII Collaboration)// Phys. Rev. Lett. 2018. V. 121. P. 081802. 